\title{
The Effect of Photoinduced Surface Oxygen Vacancies on the Charge Carrier Dynamics in $\mathrm{TiO}_{2}$ Films
}

\author{
Omur E. Dagdeviren,* Daniel Glass, Riccardo Sapienza, Emiliano Cortés, Stefan A. Maier, \\ Ivan. P. Parkin, Peter Grütter, and Raul Quesada-Cabrera*
}

Cite This: https://doi.org/10.1021/acs.nanolett.1c02853

Read Online

ACCESS | 岁 Metrics \& More | 回 Article Recommendations

S1 Supporting Information

ABSTRACT: Metal-oxide semiconductors (MOS) are widely utilized for catalytic and photocatalytic applications in which the dynamics of charged carriers (e.g., electrons, holes) play important roles. Under operation conditions, photoinduced surface oxygen vacancies (PI-SOV) can greatly impact the dynamics of charge carriers. However, current knowledge regarding the effect of PISOV on the dynamics of hole migration in MOS films, such as titanium dioxide, is solely based upon volume-averaged measurements and/or vacuum conditions. This limits the basic understanding of hole-vacancy interactions, as they are not capable of revealing time-resolved variations during operation. Here, we measured the effect of PI-SOV on the dynamics of hole migration

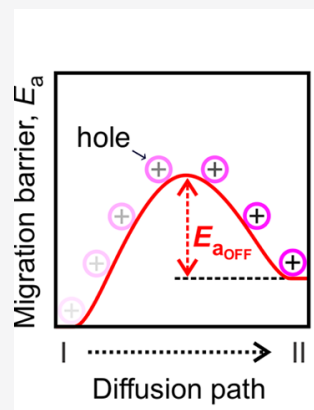

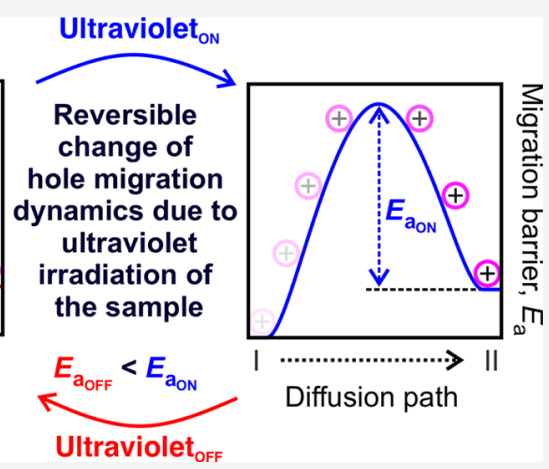
using time-resolved atomic force microscopy. Our findings demonstrate that the time constant associated with hole migration is strongly affected by PI-SOV, in a reversible manner. These results will nucleate an insightful understanding of the physics of hole dynamics and thus enable emerging technologies, facilitated by engineering hole-vacancy interactions.

KEYWORDS: Time-resolved atomic force microscopy, defected metal-oxide semiconductors, titanium dioxide $\left(\mathrm{TiO}_{2}\right)$, ultraviolet irradiation, surface defects

\section{INTRODUCTION AND BACKGROUND}

Metal-oxide semiconductors (MOS) (i.e., semiconductors consisting of a metal and oxygen) have attracted considerable interest in recent years within a wide range of industrial areas such as catalysis, ${ }^{1,2}$ electronics, ${ }^{3}$ sensing, ${ }^{4-6}$ and environmental applications. ${ }^{7,8}$ This widespread use can be explained largely by the ability to tune the properties of MOS to the desired application. A common method of achieving this without drastically changing other properties of the material, such as toxicity and biocompatibility, is through defect engineering. $3,5,6,8-11$ Specifically, oxygen vacancy $\left(V_{O}\right)$ defects can become highly reactive sites, greatly influencing the functional properties of MOS, ${ }^{8,10,12,13}$ even in very small concentrations (i.e., a few $\mathrm{ppm}^{12}$ ). Nevertheless, because of the inherent low concentrations and high reactivity of $\mathrm{V}_{\mathrm{O}}$, the probing of such states experimentally is particularly challenging, especially when using practical (e.g., polycrystalline) substrates under ambient conditions. ${ }^{13}$ As these defects play an important role in many catalytic and electronic applications, ${ }^{14}$ the study of real-time effects of $V_{O}$ on carriers within MOS is crucial. Photoinduced surface oxygen vacancies have been studied in depth over the last few decades. ${ }^{15,16}$ Recently, we investigated and reviewed the formation of photoinduced surface $V_{O}$ and showed how the dynamics of photoinduced surface $V_{O}$ could be studied through photoinduced enhanced Raman spectroscopy under photocatalytic conditions. ${ }^{4,6}$ It was demonstrated that the concentration of $\mathrm{V}_{\mathrm{O}}$ can significantly vary under ultraviolet (UV) irradiation, thereby greatly affecting the chemical reactivity of the MOS surface over time. Although many catalytic and photocatalytic processes rely heavily on the movement and interactions of charge carriers, the interactions between photoinduced surface $\mathrm{V}_{\mathrm{O}}$ and charge carriers is generally unclear. Hence, a better understanding of how the surface $V_{O}$ defects induced during photoirradiance affect the mobility and migration of carriers at the relevant time scales is needed.

In this work, we demonstrate the effect of the photoinduced surface $\mathrm{V}_{\mathrm{O}}$ on charge carrier dynamics using time-resolved atomic force microscopy (TR-AFM). Notably, these studies were performed on conventional titanium dioxide $\left(\mathrm{TiO}_{2}\right)$ films deposited using chemical vapor deposition, as they are widely

Received: July 29, 2021

Revised: September 22, 2021 
produced in the industry. Our measurements clearly show that the time constants associated with the hole migration decrease while an increase for the migration barrier is observed due to the photoinduced formation of surface $\mathrm{V}_{\mathrm{O}}$. Photoinduced surface $V_{O}$ can act as carrier trap sites, reducing the reactivity and mobility of hole carriers. With the termination of UV irradiation, a complete recovery of the hole migration barrier was observed, which can be explained by the temporary nature of photoinduced $\mathrm{V}_{\mathrm{O}}$. These results demonstrate the important effects $\mathrm{V}_{\mathrm{O}}$ generated in situ during catalysis can play, highlighting the need for further studies of defects under operation conditions.

\section{SUMMARY OF METHODS}

We employed an extended version of the time-resolved electrostatic force microscopy in our experiments that was initially established by Schirmeisen et al. ${ }^{17}$ by using fastdetection electronics and high-frequency cantilevers, details of which can be found elsewhere. ${ }^{18}$ Figure 1 summarizes the

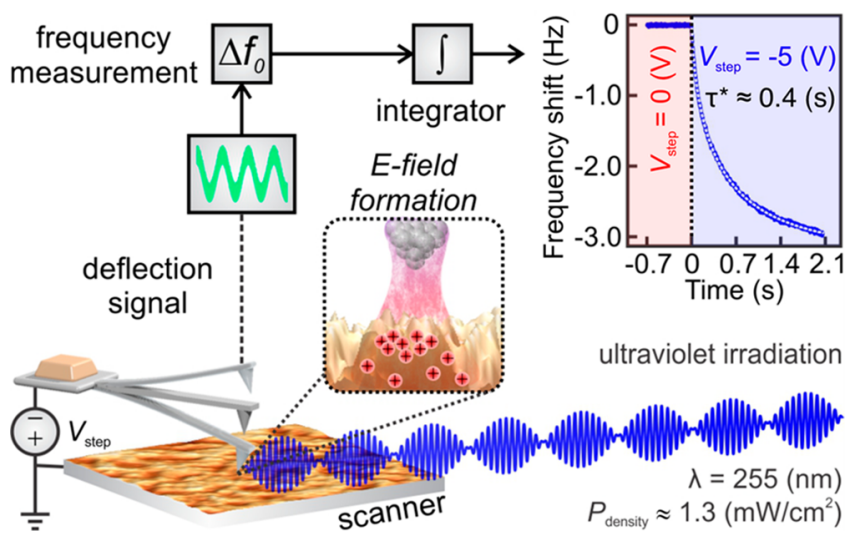

Figure 1. Schematic explanation of the experimental procedure and the local measurement of hole migration time constants as a function of temperature and UV irradiation. To investigate the effect of photoinduced surface oxygen vacancies on the charge carrier dynamics (i.e., holes) in $\mathrm{TiO}_{2}$ films, the sample is irradiated under UV light $\left(\lambda=255 \mathrm{~nm}, P_{\text {density }}=1.3 \mathrm{~mW} \mathrm{~cm}^{-2}\right)$ at ambient conditions. A bias voltage is applied between the tip and the sample $\left(V_{\text {step }}=-5\right.$ $\mathrm{V})$. The applied bias voltage results in a time-dependent Coulomb interaction between the cantilever and the sample. This additional force leads to a resonance frequency shift, $\Delta f_{0}$, which is demodulated. The time-dependent frequency shift data is used to extract the dynamics, that is, time constant $\left(\tau^{*}\right)$, and associated energy barriers of hole migration. As illustrated in the $\Delta f_{0}$ vs time plot, the tip-sample bias is applied as a step function (red highlighted region, $V_{\text {step }}=0 \mathrm{~V}$, blue highlighted region $V_{\text {step }}=-5 \mathrm{~V}$ ).

experimental procedure that we used for our experiments (see the Supporting Information for details of sample preparation, choice, and experimental details). As explained in Figure 1, applying a localized external electric field at a close proximity to the sample causes the charge carriers in the sample to move, which involves discrete hops from their initial sites to neighboring sites. As a result, the motion of the charge carriers in the material leads to a decay of the internal electric field. This time-dependent variation in the tip-sample interaction force can be assessed with the measurement of the resonance frequency shift of the oscillating cantilever, $\Delta f_{0}$. As explained in detail in the Supporting Information, the effective activation energy related with the migration of holes, $E_{\mathrm{a}}{ }^{*}$, and the migration barrier for a single hole motion, $E_{a}$, can be extracted with this information. Note that $E_{\mathrm{a}}{ }^{*}$ and $E_{\mathrm{a}}$ referred to in eq 5 of the Supporting Information does not correspond to any chemical reaction or process but rather to the effective barrier encountered by charge carriers when moving from one position to another within the material structure.

\section{RESULTS AND DISCUSSION}

An initial characterization of the $\mathrm{TiO}_{2}$ film was performed prior to TR-AFM studies using Raman spectroscopy and X-ray diffraction (see Sections 6 and 7 of the Supporting Information). Figure S2 shows the average Raman spectra of 50 randomly sampled positions across the surface. Characteristic $E_{g}$ and $A_{2 g}$ rutile modes were observed at ca. 445 and 610 $\mathrm{cm}^{-1}$, respectively. Figure S3 reveals a representative X-ray diffractogram of the $\mathrm{TiO}_{2}$ film, corresponding to the rutile phase, which we used due to its superior stability over the anatase phase under ambient conditions and having significantly fewer surface radicals (see Section 1 of the Supporting Information). ${ }^{7,19-23}$ No traces of the anatase phase were detected at any sampled position in either Raman spectra or Xray diffractograms, confirming the conversion of the asprepared film to the rutile phase.

MOS such as $\mathrm{TiO}_{2}$ are influenced by strong electric fields, such as those induced by a bias voltage applied using a scanned probe (vide supra). When the charged tip is brought close to the material surface, it can mobilize charge carriers throughout the substrate from both surface and bulk. Carriers then generally recombine within the time frame of nanoseconds in $\mathrm{TiO}_{2} ;{ }^{24-26}$ however, charge recombination rates are largely affected by the concentration of defects within the structure $^{25,27-29}$ and absorbed water. ${ }^{30}$ The interaction between charge carriers and defects strongly affects the carrier migration barrier and, therefore, the $\tau^{*}$ value measured through TR-AFM. ${ }^{31}$ The characteristics of surface and bulk charge carriers (e.g., charge dynamics, trapping ability, etc.) can largely differ. ${ }^{24,323}$ As detailed in Section 2, TR-AFM probes the sample through an applied electric field. A timeresolved measurement of the resonance frequency shift of the oscillation probe, due to the applied electric field, reflects the dynamics of charge carriers within a probing volume across the surface toward the bulk of the substrate. Therefore, TR-AFM measurements represent an average over the substrate with a probing depth determined by the shielding of the externally applied electric field within the substrate.

In $\mathrm{TiO}_{2}$, the dominant charge carriers are well-known to be electrons and holes, ${ }^{15,16,24}$ as opposed to ions, which are carriers in other materials, such as $\mathrm{LiFePO}_{4} \cdot{ }^{18}$ Carrier mobilities for different morphologies and structures of $\mathrm{TiO}_{2}$ have been widely reported in the literature and strongly depend on the material properties and defect concentration. ${ }^{11,29}$ The typical resolution of TR-AFM does not allow for the detection of electrons or the transient response of holes upon UV irradiation, that is, an instantaneous response to surface irradiation, due to their relatively short lifetimes $(<1$ $\mathrm{ms}) .{ }^{15,16,24}$ The nature and the time scale of our measurements are different than the time-dependent response of the photogenerated electrons and hole that have been studied in detail before by different researchers. ${ }^{15,16,24}$ Here, we measure the collective migration of charge carriers due to an externally applied electric field. For $\mathrm{TiO}_{2}$ films, the externally applied electric field can act upon electrons and holes, that is, the main charge carriers of the system. ${ }^{15,16,24}$ Our attempts to measure 
the dynamics of electrons on different sample systems (e.g., sapphire, gold) with an externally applied electric field illustrate that electrons have relatively short lifetimes, beyond the capability of the employed time-resolved technique. ${ }^{18,31}$ As such, it is reasonable to assume that the $\tau^{*}$ measured in our studies primarily corresponds to a hole migration, which is expected to have significantly longer lifetimes (100s of ms) in parallel to the charge carrier dynamics of similar sample systems, for example, oxygen vacancy dynamics in inorganic perovskites (see Section 3 of the Supporting Information). ${ }^{31,34}$ It is important to note that $\mathrm{UV}$-induced oxygen vacancies $\left(\mathrm{V}_{\mathrm{O}}\right)$ can be either neutral $\left(\mathrm{V}_{\mathrm{O}}{ }^{0}\right)$ or charged (e.g., $\left.\mathrm{V}_{\mathrm{O}}{ }^{+}, \mathrm{V}_{\mathrm{O}}{ }^{2+}\right){ }^{35,36}$ The dominant charge state of vacancies is determined by their formation energies and the defect density and affects the overall electrical conductivity of the film. ${ }^{31,37} \mathrm{TiO}_{2}$ films are semiconducting in nature but contain a wide band gap (ca. 3 $\mathrm{eV}$ ). Hence, we may expect that $\mathrm{V}_{\mathrm{O}}{ }^{+}$have the greatest effect on the conductivity of our sample system based on previous theoretical studies of rutile surfaces. ${ }^{35}$ In passing, it is important to note that the determination of the exact charge state of oxygen vacancies does not play an important role in either our experimental observations or the interpretation of those observations, but it only becomes important when experimental results are compared with ab initio calculations. ${ }^{31}$

Typically, FM-AFM studies are conducted under high vacuum conditions in order to obtain better signal-to-noise ratios (see Section 3 of the Supporting Information for details). ${ }^{38}$ Nevertheless, the generation of photoinduced surface $\mathrm{V}_{\mathrm{O}}$, in a photocatalytic MOS, such as $\mathrm{TiO}_{2}$, has been shown to not occur under vacuum conditions. ${ }^{39,40} \mathrm{~A}$ more complete understanding for this was presented by Thompson et al. ${ }^{28}$ and is briefly discussed below. Therefore, to study photoinduced $\mathrm{V}_{\mathrm{O}}$ on the dynamics of hole migration, measurements must be taken under ambient conditions, that is, under the existence of $\mathrm{H}_{2} \mathrm{O}$ and $\mathrm{O}_{2}$. However, both the sensitivity of FM-AFM measurements and the contaminants in the air may, in theory, impede the measurement of the desired physical phenomenon. For this reason, a systematic comparison of ambient and vacuum measurements is essential to ensure that the measurement is not dominated by artifacts under ambient conditions (see Section 3 of the Supporting Information for details).

Figure 2 shows a comparison of effective activation energy, $E_{\mathrm{a}}{ }^{*}$, under both high vacuum and ambient conditions. As explained above (vide supra), time-dependent frequency shift data are used to extract the dynamics of hole migration, $\tau^{*}$, and associated energy barriers for their movement. The slope of the natural logarithm of the time constant (in ms) versus $1 /\left(k_{\mathrm{B}} T\right)$ (Arrhenius plot) - where $k_{\mathrm{B}}$ is the Boltzmann constant and $T$ is the temperature in Kelvin-discloses $E_{\mathrm{a}}{ }^{*}$ corresponding to hole migration ${ }^{18}$ for high vacuum (black curve) and ambient (blue curve) measurements. $E_{\mathrm{a}}{ }^{*}$ values were determined as $110 \pm 5$ and $107 \pm 8 \mathrm{meV}$ for the same measurement area under vacuum and ambient conditions, respectively (see Section 3 of the Supporting Information). The corresponding single-hole migration barriers, $E_{\mathrm{a}}$, of vacuum and ambient measurements are $80 \pm 5$ and $75 \pm 6 \mathrm{meV}$, respectively. These results demonstrate the absence of a statistical difference between vacuum and ambient measurements, which confirms that a TR-AFM analysis can be performed under ambient conditions needed for photoinduced vacancy production using high-energy UV photons $(\lambda=255 \mathrm{~nm})$.

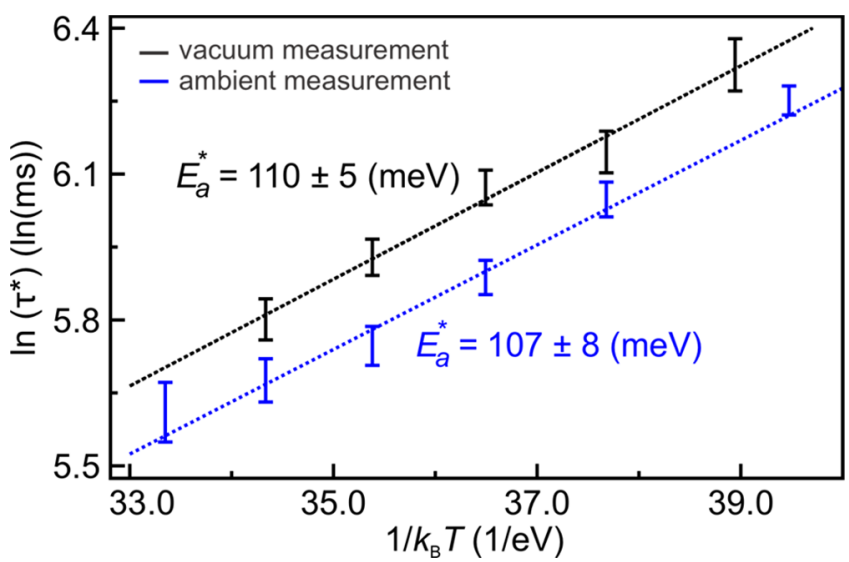

Figure 2. Measurement of effective activation barrier of holes, $E_{\mathrm{a}}{ }^{*}$, in $\mathrm{TiO}_{2}$ films under high vacuum (black curve) and ambient conditions (blue curve), respectively. The $E_{\mathrm{a}}{ }^{*}$ is obtained from the slope of the Arrhenius plot of the natural logarithm of the time constant (in ms) vs $1 /\left(k_{\mathrm{B}} T\right)$, where $k_{\mathrm{B}}$ is the Boltzmann constant, and $T$ is the temperature in Kelvin. $E_{\mathrm{a}}{ }^{*}$ values were calculated to be $110 \pm 5$ and $107 \pm 8 \mathrm{meV}$ for vacuum and ambient conditions, respectively, which means less than $2 \%$ variation. This agreement thus shows that TR-AFM measurements can be reproducibly conducted under ambient conditions. In this figure, vertical bars show the variation of the time constant across the surface within an area of $1000 \mathrm{~nm} \times$ $1000 \mathrm{~nm}$ (see Section 3 of the Supporting Information for further details).

After an analysis under ambient conditions, further measurements were conducted under UV irradiation. $\mathrm{TiO}_{2}$ is a wide band gap semiconductor $\left(E_{\mathrm{bg}}>3 \mathrm{eV}\right)^{11,29,32}$ and absorbs photons within the UV region $(<375 \mathrm{~nm})$. Therefore, irradiating $\mathrm{TiO}_{2}$ with high-energy UV light $(\lambda=255 \mathrm{~nm})$ can significantly affect both charge carrier density and defect concentration within the substrate. The behavior of carriers and defects has been shown to vary the surface and bulk of materials. Therefore, we first discuss the location in which photoinduced defects occur within the substrate and their interactions with charge carriers. Typical $\mathrm{TiO}_{2}$ films produced via aerosol-assisted chemical vapor deposition under the conditions used in this study are on the order of a few hundred nanometers thick. ${ }^{41}$ The penetration depth of highenergy UV irradiation is commonly estimated within 10-30 $\mathrm{nm},{ }^{42}$ although some studies have claimed UV absorption across larger depths. ${ }^{16,43,44}$ On the basis of literature reports ${ }^{45,46}$ and the particular physical properties of our films, we estimated an absorption penetration of ca. $17 \mathrm{~nm}$ in our case. Nevertheless, while some UV photons may reach the bulk of the material and lead to the formation of charge carriers, the dominant changes in carrier concentration and carrier lifetime occur at the surface. First, carrier recombination strongly depends on both the position at which the carriers are produced-either at the surface or within the bulk-and the concentration of defects in the substrate, where surface carriers tend to have slow recombination rates. ${ }^{2,24,28,32,33,47}$ Second, the mechanism by which photoinduced defects are generated is generally understood to be mediated through adsorbed molecules and radicals in contrast to a direct photolysis of $\mathrm{Ti}-\mathrm{O}$ bonds. ${ }^{28,48}$ This can only occur at the surface, where adsorbed species are found. Third, induced carriers also tend to migrate from the bulk to the surface over time. ${ }^{28,33}$ Hole traps within the bulk can quickly saturate under a critical photon flux, allowing generated holes to efficiently reach the 
surface. $^{49}$ Finally, surface bridging oxygen atoms have fewer neighboring $\mathrm{Ti}$ atoms than bulk oxygen atoms due to their position within the lattice; hence, the former require less energy to form $\mathrm{V}_{\mathrm{O}}$. Photoinduced holes can also greatly weaken $\mathrm{Ti}-\mathrm{O}$ bonds, thereby making the surface bridging oxygen even more susceptible to be removed, increasing the probability of a $\mathrm{V}_{\mathrm{O}}$ defect formation. Hence, although bulk carriers can be trapped and weaken bonds within the crystal, the formation of defects in the bulk is unlikely under UV irradiation. Instead, the primary changes in photoinduced carrier concentration and carrier lifetimes will take place at the surface, where additional surface defects can be induced.

To investigate the effect of UV irradiation on $\tau^{*}$, measurements were then conducted at a single position over a period of time by employing an active drift control. The sample was left in the dark for a long period of time (i.e., more than $5 \mathrm{~h}$ ) to measure the stability of $\tau^{*}$ over time under ambient conditions. Figure 3 shows that, after the same

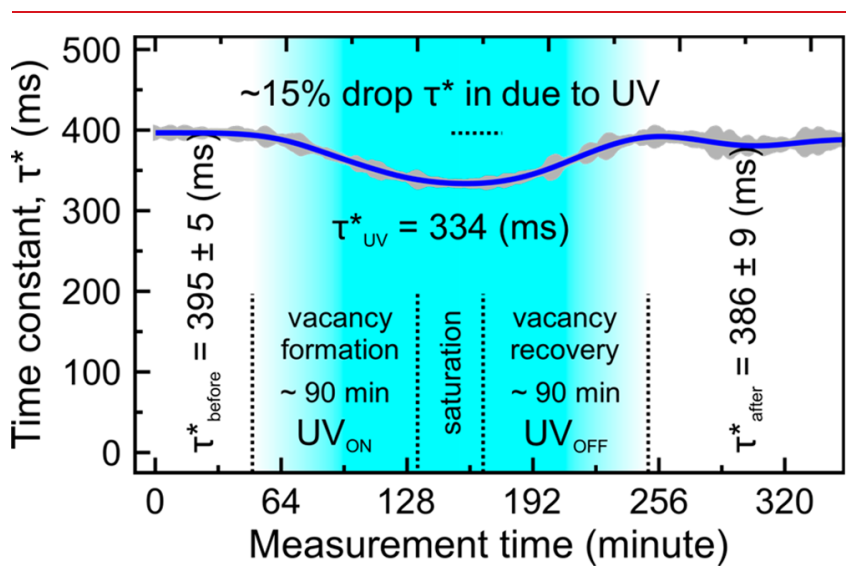

Figure 3. Change in time constant of hole migration barrier, $\tau^{*}$, upon UV irradiation, measured at a single position on $\mathrm{TiO}_{2}$. These measurements were performed initially in the dark and, subsequently, under a UV exposure followed by another period in the dark. The vacancy formation saturated within $\sim 90 \mathrm{~min}$. Upon the termination of UV exposure, the surface recovery was observed within $\sim 90 \mathrm{~min}$ as well. The conducting of experiments at the same location and the same tip-sample separation requires a very stable and low-drift microscope with an active drift compensation, which was achieved by a Gnome X Scanning Microscopy control module. Repeated experiments were conducted with a rolling average of 15 measurements to enhance the signal-to-noise ratio and measured $\tau^{*}$ (blue curve) with less than $3 \%$ uncertainty. ${ }^{31}$ Therefore, the $15 \%$ drop and reversible change in $\tau^{*}$ was directly attributed to the influence of UV absorption. The gray regions in the figure illustrate the standard deviation for each data point.

position was continuously sampled in the dark over $\sim 1 \mathrm{~h}$, no significant variation of $\tau^{*}(395 \pm 5 \mathrm{~ms})$ was observed. After this period, a steady drop in $\tau^{*}$ was found over an irradiation period of $\sim 90 \mathrm{~min}$, until an effective minimum was reached $\left(\tau^{*} \approx 334 \mathrm{~ms}\right.$, i.e., $15 \%$ decrease $)$. This decrease in $\tau^{*}$ was recovered $(386 \pm 9 \mathrm{~ms})$ within a similar period of time once the UV source was switched off. The recovery of the time constant associated with hole migration indicates a completely reversible process. The variation of $\tau^{*}$ at a single temperature is not informative to assess the variation of $E_{\mathrm{a}}{ }^{*}$, as $E_{\mathrm{a}}{ }^{*}$ can only be calculated by the slope of the Arrhenius plot of the natural logarithm of the time constant (see Section 5 of the Supporting Information for details). For this reason, with the same procedure while the sample temperature was tuned, the average $E_{\mathrm{a}}{ }^{*}$ value showed a significant increase, from $107 \pm 8$ to $189 \pm 10 \mathrm{meV}$ after it stabilized under UV irradiation. Also, the corresponding single-hole activation barrier, $E_{a}$ increased from $75 \pm 6$ to $135 \pm 8 \mathrm{meV}$ upon UV irradiation (see Section 5 of the Supporting Information for details).

As discussed above (vide supra), the primary change within $\mathrm{TiO}_{2}$ under UV irradiation is the increased concentration of surface $\mathrm{V}_{\mathrm{O}}$ defect states. While from a chemical perspective an increased defect concentration is often related with faster reaction times and, therefore, lower activation energies, it is important to note that, here, we defined $E_{\mathrm{a}}$ and $E_{\mathrm{a}}{ }^{*}$ not in the chemical sense but as the effective energy barrier a charge carrier experiences when moving from one position to another within the lattice. Hence, an increase in $E_{\mathrm{a}}$ indicates that the hole migration within $\mathrm{TiO}_{2}$ is impeded due to additional surface $\mathrm{V}_{\mathrm{O}}$ formed under $\mathrm{UV}$ irradiation. Also, a decrease in hole mobility is implied with an increase in $E_{\mathrm{a}}$ due to the relation with activation energy and diffusion, the derivation of which has been explained in detail elsewhere and can be presented as follows. ${ }^{31}$

$$
\mu=\frac{q n l^{2}}{2 \alpha h} \exp \left(\frac{-E_{\mathrm{a}}}{k_{\mathrm{B}} T}\right)
$$

In eq $1, \mu$ is the mobility of the hole, $q$ is the charge, $l$ is the distance between hole configurations, $\alpha$ is the dimensionality of the process, $h$ is Planck's constant, $k_{\mathrm{B}}$ is the Boltzmann constant, and $T$ is the system temperature. The increase in $E_{\mathrm{a}}$ can be explained by an understanding of factors that affect the hole mobility and the interaction between surface $V_{O}$ and holes, as presented by eq 1 . It is worth noting that the effect of $\tau^{*}$ on $\mu$ is included by the dependence of $E_{\mathrm{a}}$ on $\tau^{*}$ (see Section 2 of the Supporting Information) and hopping rate, time, and distance terms utilized to derive eq 1 (see the Supporting Information of ref 31 for the details of the relation between $\mu$ and $E_{\mathrm{a}}$ ). The position of the defects has also been shown to either promote recombination (bulk defects) or enhance carrier separation (surface defects). ${ }^{32,33,50}$ As eq 1 shows, both recombination and, conversely, carrier separation, have a significant impact in carrier mobility-since an increased carrier separation is often accompanied by an increase in mobility. One may therefore expect an increase in carrier mobility, that is, a decrease in $E_{a}$, with an increased surface $V_{O}$ concentration, as the mobility and $E_{\mathrm{a}}$ are inversely related according to eq 1 . The increase in $E_{\mathrm{a}}$ upon UV irradiation is thus rather surprising. Induced carriers can be trapped in shallow (low energy) traps, ${ }^{13,51}$ which can affect carrier lifetime, but it is also expected to affect the carrier mobility. At temperatures close to room temperature, holes will rapidly recombine or migrate to more stable trapping sites ${ }^{52}$ and localize around defect sites. $^{53}$ Surface-bridging oxygen and oxygen vacancy defects are considered to act as deep (highenergy) traps, thus greatly affecting the mobility of charge carriers by potentially altering the dimensionality of the hole migration (presented by the $\alpha$ term in eq 1 ), that is, by affecting the hole migration deep into the bulk. ${ }^{47,51,54-56}$ Once holes are trapped at a lattice site, their reactivity has been found to decrease, thereby increasing their lifetime $e^{2,24,47}$ while also significantly reducing their mobility. Besides, researchers have recently highlighted the important role polarons may play in the trapping of other charge carriers and charge carrier transport in $\mathrm{TiO}_{2}{ }^{53}$ For rutile $\mathrm{TiO}_{2}$, the self-trap energy for 
polaronic hole formation was found to be positive, which suggests the hole is localized at a lattice site, ${ }^{49}$ for example, a bridging oxygen or oxygen vacancy. Although the interaction between a charge carrier and a vacancy or trap state can vary considerably, many reported interactions indicate a reduced carrier mobility as a result of the interaction. ${ }^{53,54,57,58}$ Interactions of this nature have also been shown to occur in other forms of $\mathrm{TiO}_{2}$ and other materials. ${ }^{51,59,60}$ Hence, the mobility of induced holes can largely be impeded by the presence of photoinduced $\mathrm{V}_{\mathrm{O}}$ defects, thus resulting in an increase in $E_{\mathrm{a}}^{*}$, as observed in the current study. These photoinduced surface vacancies are only temporary under ambient conditions and will subsequently heal after the UV source is switched off, ${ }^{6,61}$ which explains the reversible behavior of the $\tau^{*}$ curve in Figure 3 .

\section{CONCLUSION}

Changes in hole carrier relaxation lifetimes and associated migration barriers were monitored in $\mathrm{TiO}_{2}$ films under highenergy UV irradiation at ambient conditions. By measuring the relaxation lifetime at different temperatures, an effective activation energy of the migration of a single hole $\left(E_{\mathrm{a}}\right)$ was determined, allowing for insight into the mobility of induced carriers within the crystal lattice. A stable relaxation lifetime was observed under dark conditions that subsequently was found to reversibly decrease after an exposure to UV light. A complete recovery of the initial carrier lifetimes was observed after the removal of the UV irradiation. The UV treatment also resulted in an $\sim 45 \%$ increase in $E_{a}$, which indicates a notable reduction of hole mobility. This surprising observation was assigned to an increase in hole trapping due to interactions with photoinduced surface $V_{O}$ sites. Once the UV source is switched off, induced vacancy states heal under ambient conditions, reducing the time constants associated with the migration of holes to its original value. Surface vacancies are preferred adsorption sites for many molecules, and therefore the trapping of hole carriers may be highly beneficial for reducing chemistry and photocatalytic applications. As such, these findings are strongly relevant to the design and understanding of emerging catalytic and photocatalytic systems that rely upon the migration and mobility of charge carriers in MOS. We believe they also account for the scientific basis of future time-resolved scanning probe microscopy experiments that involve the critical role of UV-induced surface oxygen vacancies in these applications.

\section{ASSOCIATED CONTENT}

\section{(s) Supporting Information}

The Supporting Information is available free of charge at https://pubs.acs.org/doi/10.1021/acs.nanolett.1c02853.

Sample preparation; time-resolved atomic force microscopy; measurements under ambient conditions and comparisons with different samples; ultraviolet irradiation of the sample; additional discussion on the effect of ultraviolet irradiation of the sample; Raman spectroscopy measurements; X-ray diffraction measurements (PDF)

\section{AUTHOR INFORMATION}

\section{Corresponding Authors}

Omur E. Dagdeviren - Department of Mechanical

Engineering, Ecole de technologie supérieure, University of
Quebec, Montreal H3C 1K3 Quebec, Canada; ○ orcid.org/ 0000-0002-4881-9280; Email: omur.dagdeviren@ etsmtl.ca

Raul Quesada-Cabrera - Department of Chemistry, University College London, London WC1H OAJ, U.K.; Fotoelectrocatálisis para Aplicaciones Medioambientales, Departamento de Química, Universidad de Las Palmas de Gran Canaria, Las Palmas de Gran Canaria 35017, Spain; Email: r.quesada@ucl.ac.uk

\section{Authors}

Daniel Glass - The Blackett Laboratory, Department of Physics, Imperial College London, London SW7 2AZ, U.K.; Department of Chemistry, University College London,

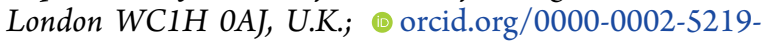
4148

Riccardo Sapienza - The Blackett Laboratory, Department of Physics, Imperial College London, London SW7 2AZ, U.K.; - orcid.org/0000-0002-4208-0374

Emiliano Cortés - Chair in Hybrid Nanosystems, Faculty of Physics, Ludwig Maximilians Universität München, München 80539, Germany; 이이이.org/0000-0001-8248-4165

Stefan A. Maier - The Blackett Laboratory, Department of Physics, Imperial College London, London SW7 2AZ, U.K.; Chair in Hybrid Nanosystems, Faculty of Physics, Ludwig Maximilians Universität München, München 80539,

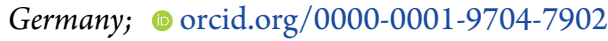

Ivan. P. Parkin - Department of Chemistry, University College

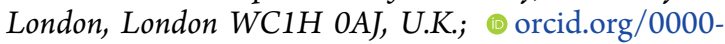
0002-4072-6610

Peter Grütter - Department of Physics, McGill University, Montreal H3A 2T8 Quebec, Canada; (1) orcid.org/00000003-1719-8239

Complete contact information is available at:

https://pubs.acs.org/10.1021/acs.nanolett.1c02853

\section{Author Contributions}

O.E.D. designed and conducted time-resolved atomic force microscopy experiments and analyzed the data. D.G. prepared the film and helped O.E.D. with the initial setup of experiments. O.E.D. prepared figures. D.G. performed the characterization of the film with Raman spectroscopy and Xray diffraction measurements and prepared corresponding supplemental figures. O.E.D. and D.G. wrote the manuscript. All authors participated in the analysis and the interpretation of the data and commented on the manuscript.

\section{Notes}

The authors declare no competing financial interest.

\section{ACKNOWLEDGMENTS}

This work was supported by the Natural Sciences and Engineering Research Council of Canada and Le Fonds de Recherche du Québec - Nature et Technologies. O.E.D. also gratefully acknowledges funds provided by École de technologie supérieure, University of Québec. D.G. acknowledges funding from the UK MOD for the Ph.D. under Contract No. DSTLX-1000116630. R.Q.C. would thanks the Beatriz Galindo Program, Ministerio de Educación y Formación Profesional, Spain. S.A.M. and E.C. acknowledge funding and support from the Deutsche Forschungsgemeinschaft (DFG, German Research Foundation) under Germany's Excellence Strategy-EXC 2089/1-390776260, the Bavarian 
program Solar Energies Go Hybrid (SolTech), the Center for NanoScience (CeNS). S.A.M. additionally acknowledges the Lee-Lucas Chair in Physics. E.C. acknowledges the European Commission through the ERC Starting Grant CATALIGHT (802989). I.P.P. acknowledges support from EPSRC Centre of Doctoral Training in Molecular Modelling and Material Science under Grant No. EP/L015862/1. The authors also acknowledge support from EPSRC-UK under Grant No. EP/ M013812/1, Reactive Plasmonics. The authors acknowledge financial support from the EPSRC Grant No. EP/R034540/1 for the JSPS-EPSRC-McGill University collaboration on "Defect Functionalized Sustainable Energy Materials: From Design to Devices Application”.

\section{REFERENCES}

(1) Kiriakidis, G.; Binas, V. Metal Oxide Semiconductors as Visible Light Photocatalysts. J. Korean Phys. Soc. 2014, 65 (3), 297-302.

(2) Linsebigler, A. L.; Lu, G.; Yates, J. T. Photocatalysis on $\mathrm{TiO}_{2}$ Surfaces: Principles, Mechanisms, and Selected Results. Chem. Rev. 1995, 95, 735-758.

(3) Zeng, Y.; Lai, Z.; Han, Y.; Zhang, H.; Xie, S.; Lu, X. OxygenVacancy and Surface Modulation of Ultrathin Nickel Cobaltite Nanosheets as a High-Energy Cathode for Advanced Zn-Ion Batteries. Adv. Mater. 2018, 30 (33), 1802396.

(4) Ben-Jaber, S.; Peveler, W. J.; Quesada-Cabrera, R.; Cortés, E.; Sotelo-Vazquez, C.; Abdul-Karim, N.; Maier, S. A.; Parkin, I. P. Photo-Induced Enhanced Raman Spectroscopy for Universal UltraTrace Detection of Explosives, Pollutants and Biomolecules. Nat. Commun. 2016, 7, 12189.

(5) Wu, H.; Wang, H.; Li, G. Metal Oxide Semiconductor SERSActive Substrates by Defect Engineering. Analyst 2017, 142, 326.

(6) Glass, D.; Cortés, E.; Ben-Jaber, S.; Brick, T.; Peveler, W. J. W. J.; Blackman, C. S. C. S.; Howle, C. R.; Quesada-Cabrera, R.; Parkin, I. P. I. P.; Maier, S. A. S. A. Dynamics of Photo-Induced Surface Oxygen Vacancies in Metal-Oxide Semiconductors Studied Under Ambient Conditions. Adv. Sci. 2019, 6 (22), 1901841.

(7) Hanaor, D. A. H.; Sorrell, C. C. Review of the Anatase to Rutile Phase Transformation. J. Mater. Sci. 2011, 46 (4), 855-874.

(8) Schweke, D.; Mordehovitz, Y.; Halabi, M.; Shelly, L.; Hayun, S. Defect Chemistry of Oxides for Energy Applications. Adv. Mater. 2018, 30 (41), 1706300.

(9) Zhou, Y.; Zhang, Z.; Fang, Z.; Qiu, M.; Ling, L.; Long, J.; Chen, L.; Tong, Y.; Su, W.; Zhang, Y.; Wu, J. C. S.; Basset, J. M.; Wang, X.; $\mathrm{Yu}, \mathrm{G}$. Defect Engineering of Metal-Oxide Interface for Proximity of Photooxidation and Photoreduction. Proc. Natl. Acad. Sci. U. S. A. 2019, 116 (21), 10232-10237.

(10) Xiong, L.-B.; Li, J.-L.; Yang, B.; Yu, Y. Ti 3+ in the Surface of Titanium Dioxide: Generation, Properties and Photocatalytic Application. J. Nanomater. 2012, 2012, 831524.

(11) Bai, S.; Zhang, N.; Gao, C.; Xiong, Y. Defect Engineering in Photocatalytic Materials. Nano Energy 2018, 53, 296-336.

(12) Gunkel, F.; Christensen, D. V.; Chen, Y. Z.; Pryds, N. Oxygen Vacancies: The (in)Visible Friend of Oxide Electronics. Appl. Phys. Lett. 2020, 116 (12), 120505.

(13) Pacchioni, G. Oxygen Vacancy: The Invisible Agent on Oxide Surfaces. ChemPhysChem 2003, 4 (10), 1041-1047.

(14) Hüttenhofer, L.; Eckmann, F.; Lauri, A.; Cambiasso, J.; Pensa, E.; Li, Y.; Cortés, E.; Sharp, I. D.; Maier, S. A. Anapole Excitations in Oxygen-Vacancy-Rich $\mathrm{TiO}_{2-\mathrm{x}}$ Nanoresonators: Tuning the Absorption for Photocatalysis in the Visible Spectrum. ACS Nano 2020, 14 (2), $2456-2464$

(15) Fujishima, A.; Zhang, X.; Tryk, D. A. TiO 2 Photocatalysis and Related Surface Phenomena. Surf. Sci. Rep. 2008, 63 (12), 515-582.

(16) Schneider, J.; Matsuoka, M.; Takeuchi, M.; Zhang, J.; Horiuchi, Y.; Anpo, M.; Bahnemann, D. W. Understanding $\mathrm{TiO}_{2}$ Photocatalysis: Mechanisms and Materials. Chem. Rev. 2014, 114 (19), 9919-9986.
(17) Schirmeisen, A.; Taskiran, A.; Fuchs, H.; Roling, B.; Murugavel, S.; Bracht, H.; Natrup, F. Probing Ion Transport at the Nanoscale: Time-Domain Electrostatic Force Spectroscopy on Glassy Electrolytes. Appl. Phys. Lett. 2004, 85 (11), 2053-2055.

(18) Mascaro, A.; Wang, Z.; Hovington, P.; Miyahara, Y.; Paolella, A.; Gariepy, V.; Feng, Z.; Enright, T.; Aiken, C.; Zaghib, K.; Bevan, K. H.; Grutter, P. Measuring Spatially Resolved Collective Ionic Transport on Lithium Battery Cathodes Using Atomic Force Microscopy. Nano Lett. 2017, 17 (7), 4489-4496.

(19) Zhang, H.; Banfield, J. F. Thermodynamic Analysis of Phase Stability of Nanocrystalline Titania Reactivity of Iron Oxyhydroxide Nanoparticles View Project Bacterial Biomineralization View Project. J. Mater. Chem. 1998, 8 (9), 2073-2076.

(20) Smith, S. J.; Stevens, R.; Liu, S.; Li, G.; Navrotsky, A.; BoerioGoates, J.; Woodfield, B. F. Heat Capacities and Thermodynamic Functions of $\mathrm{TiO}_{2}$ Anatase and Rutile: Analysis of Phase Stability. Am. Mineral. 2009, 94 (2-3), 236-243.

(21) Sclafani, A.; Herrmann, J. M. Comparison of the Photoelectronic and Photocatalytic Activities of Various Anatase and Rutile Forms of Titania in Pure Liquid Organic Phases and in Aqueous Solutions. J. Phys. Chem. 1996, 100 (32), 13655-13661.

(22) Augustynski, J. The Role of the Surface Intermediates in the Photoelectrochemical Behaviour of Anatase and Rutile $\mathrm{TiO}_{2}$. Electrochim. Acta 1993, 38 (1), 43-46.

(23) Muscat, J.; Swamy, V.; Harrison, N. M. First-Principles Calculations of the Phase Stability of $\mathrm{TiO}_{2}$. Phys. Rev. B: Condens. Matter Mater. Phys. 2002, 65, 224112.

(24) Rothenberger, G.; Moser, J.; Graetzel, M.; Serpone, N.; Sharma, D. K. Charge Carrier Trapping and Recombination Dynamics in Small Semiconductor Particles. J. Am. Chem. Soc. 1985, 107, 8054-8059.

(25) Qian, R.; Zong, H.; Schneider, J.; Zhou, G.; Zhao, T.; Li, Y.; Yang, J.; Bahnemann, D. W.; Pan, J. H. Charge Carrier Trapping, Recombination and Transfer during $\mathrm{TiO}_{2}$ Photocatalysis: An Overview. Catal. Today 2019, 335, 78-90.

(26) Fujihara, K.; Izumi, S.; Ohno, T.; Matsumura, M. TimeResolved Photoluminescence of Particulate $\mathrm{TiO}_{2}$ Photocatalysts Suspended in Aqueous Solutions. J. Photochem. Photobiol., A 2000, 132, 99-104.

(27) Xiao, F.; Zhou, W.; Sun, B.; Li, H.; Qiao, P.; Ren, L.; Zhao, X.; $\mathrm{Fu}, \mathrm{H}$. Engineering Oxygen Vacancy on Rutile $\mathrm{TiO}_{2}$ for Efficient Electron-Hole Separation and High Solar-Driven Photocatalytic Hydrogen Evolution. Sci. China Mater. 2018, 61 (6), 822.

(28) Thompson, T. L.; Yates, J. T. Surface Science Studies of the Photoactivation of $\mathrm{TiO}_{2}-\mathrm{New}$ Photochemical Processes. Chem. Rev. 2006, 106, 4428.

(29) Rahimi, N.; Pax, R. A.; Gray, E. M. A. Review of Functional Titanium Oxides. I: $\mathrm{TiO}_{2}$ and Its Modifications. Prog. Solid State Chem. 2016, 44 (3), 86-105.

(30) Litke, A.; Su, Y.; Tranca, I.; Weber, T.; Hensen, E. J. M.; Hofmann, J. P. Role of Adsorbed Water on Charge Carrier Dynamics in Photoexcited $\mathrm{TiO}_{2}$. J. Phys. Chem. C 2017, 121 (13), 7514-7524.

(31) Dagdeviren, O. E.; Mascaro, A.; Yuan, S.; Shirani, J.; Bevan, K. H.; Grütter, P. Ergodic and Nonergodic Dynamics of Oxygen Vacancy Migration at the Nanoscale in Inorganic Perovskites. Nano Lett. 2020, 20 (10), 7530-7535.

(32) Pan, X.; Yang, M.-Q.; Fu, X.; Zhang, N.; Xu, Y.-J. Defective $\mathrm{TiO}_{2}$ with Oxygen Vacancies: Synthesis, Properties and Photocatalytic Applications. Nanoscale 2013, 5 (9), 3601.

(33) Kong, M.; Li, Y.; Chen, X.; Tian, T.; Fang, P.; Zheng, F.; Zhao, $\mathrm{X}$. Tuning the Relative Concentration Ratio of Bulk Defects to Surface Defects in $\mathrm{TiO}_{2}$ Nanocrystals Leads to High Photocatalytic Efficiency. J. Am. Chem. Soc. 2011, 133 (41), 16414-16417.

(34) Liu, C.; Wu, P.; Wu, J.; Hou, J.; Bai, H.; Liu, Z. Effective Protect of Oxygen Vacancies in Carbon Layer Coated Black $\mathrm{TiO}_{2-\mathrm{x}} /$ CNNS Hetero-Junction Photocatalyst. Chem. Eng. J. 2019, 359, 58.

(35) Wang, S. G.; Wen, X. D.; Cao, D. B.; Li, Y. W.; Wang, J.; Jiao, H. Formation of Oxygen Vacancies on the $\mathrm{TiO}_{2}\left(\begin{array}{lll}1 & 1 & 0\end{array}\right)$ Surfaces. Surf. Sci. 2005, 577 (1), 69-76. 
(36) Deák, P.; Aradi, B.; Frauenheim, T. Quantitative Theory of the Oxygen Vacancy and Carrier Self-Trapping in $\mathrm{Bulk} \mathrm{TiO}_{2}$. Phys. Rev. B: Condens. Matter Mater. Phys. 2012, 86 (19), 1-8.

(37) Janotti, A.; Varley, J. B.; Choi, M.; Van de Walle, C. G. Vacancies and Small Polarons in $\mathrm{SrTiO}_{3}$. Phys. Rev. B: Condens. Matter Mater. Phys. 2014, 90 (8), 85202.

(38) Albrecht, T. R.; Grütter, P.; Horne, D.; Rugar, D. Frequency Modulation Detection Using High-Q Cantilevers for Enhanced Force Microscope Sensitivity. J. Appl. Phys. 1991, 69 (2), 668-673.

(39) Mezhenny, S.; Maksymovych, P.; Thompson, T. L.; Diwald, O.; Stahl, D.; Walck, S. D.; Yates, J. T. STM Studies of Defect Production on the $\mathrm{TiO}_{2}(110)-(1 \times 1)$ and $\mathrm{TiO}_{2}(110)-(1 \times 2)$ Surfaces Induced by UV Irradiation. Chem. Phys. Lett. 2003, 369 (1), 152-158.

(40) Zubkov, T.; Stahl, D.; Thompson, T. L.; Panayotov, D.; Diwald, O.; Yates, J. T. Ultraviolet Light-Induced Hydrophilicity Effect on $\mathrm{TiO}_{2}(110)(1 \times 1)$. Dominant Role of the Photooxidation of Adsorbed Hydrocarbons Causing Wetting by Water Droplets. J. Phys. Chem. B 2005, 109 (32), 15454-15462.

(41) Edusi, C.; Hyett, G.; Sankar, G.; Parkin, I. P. Aerosol-Assisted CVD of Titanium Dioxide Thin Films from Methanolic Solutions of Titanium Tetraisopropoxide; Substrate and Aerosol-Selective Deposition of Rutile or Anatase. Chem. Vap. Deposition 2011, 17 (1-3), $30-36$.

(42) Stevanovic, A.; Büttner, M.; Zhang, Z.; Yates, J. T. Photoluminescence of $\mathrm{TiO}_{2}$ : Effect of UV Light and Adsorbed Molecules on Surface Band Structure. J. Am. Chem. Soc. 2012, 134 (1), 324-332.

(43) Zhang, J.; Wang, J.; Zhao, Z.; Yu, T.; Feng, J.; Yuan, Y.; Tang, Z.; Liu, Y.; Li, Z.; Zou, Z. Reconstruction of the (001) Surface of $\mathrm{TiO}_{2}$ Nanosheets Induced by the Fluorine-Surfactant Removal Process under UV-Irradiation for Dye-Sensitized Solar Cells. Phys. Chem. Chem. Phys. 2012, 14 (14), 4763-4769.

(44) Wahl, A.; Augustynski, J. Charge Carrier Transport in Nanostructured Anatase $\mathrm{TiO}_{2}$ Films Assisted by the Self-Doping of Nanoparticles. J. Phys. Chem. B 1998, 102 (40), 7820-7828.

(45) Tanabe, I.; Ozaki, Y. Far- and Deep-Ultraviolet Spectroscopic Investigations for Titanium Dioxide: Electronic Absorption, Rayleigh Scattering, and Raman Spectroscopy. J. Mater. Chem. C 2016, 4 (33), 7706-7717.

(46) Siefke, T.; Kroker, S.; Pfeiffer, K.; Puffky, O.; Dietrich, K.; Franta, D.; Ohlídal, I.; Szeghalmi, A.; Kley, E. B.; Tünnermann, A. Materials Pushing the Application Limits of Wire Grid Polarizers Further into the Deep Ultraviolet Spectral Range. Adv. Opt. Mater. 2016, 4 (11), 1780-1786.

(47) Nam, Y.; Li, L.; Lee, J. Y.; Prezhdo, O. V. Strong Influence of Oxygen Vacancy Location on Charge Carrier Losses in Reduced $\mathrm{TiO}_{2}$ Nanoparticles. J. Phys. Chem. Lett. 2019, 10, 2676-2683.

(48) Lun Pang, C.; Lindsay, R.; Thornton, G. Chemical Reactions on Rutile $\mathrm{TiO}_{2}$ (110). Chem. Soc. Rev. 2008, 37, 2328-2353.

(49) Thompson, T. L.; Yates, J. T. Monitoring Hole Trapping in Photoexcited $\mathrm{TiO}_{2}(110)$ Using a Surface Photoreaction. J. Phys. Chem. B 2005, 109 (39), 18230-18236.

(50) Zhuang, J.; Dai, W.; Tian, Q.; Li, Z.; Xie, L.; Wang, J.; Liu, P.; Shi, X.; Wang, D. Photocatalytic Degradation of RhB over $\mathrm{TiO}_{2}$ Bilayer Films: Effect of Defects and Their Location. Langmuir 2010, 26 (12), 9686-9694.

(51) Morgan, B. J.; Watson, G. W. Polaronic Trapping of Electrons and Holes by Native Defects in Anatase $\mathrm{TiO}_{2}$. Phys. Rev. B: Condens. Matter Mater. Phys. 2009, 80 (23), 233102.

(52) Yang, S.; Brant, A. T.; Halliburton, L. E. Photoinduced SelfTrapped Hole Center in $\mathrm{TiO}_{2}$ Crystals. Phys. Rev. B: Condens. Matter Mater. Phys. 2010, 82 (3), No. 035209.

(53) Liu, B.; Zhao, X.; Yu, J.; Parkin, I. P.; Fujishima, A.; Nakata, K. Intrinsic Intermediate Gap States of $\mathrm{TiO}_{2}$ Materials and Their Roles in Charge Carrier Kinetics. J. Photochem. Photobiol., C 2019, 39, 1-57.

(54) Zawadzki, P. Semiconductor Photocatalysis: Electronic Hole Trapping in $\mathrm{TiO}_{2}, \mathrm{PhD}$ Thesis, Technical University of Denmark, 2011.
(55) Berger, T.; Sterrer, M.; Diwald, O.; Knözinger, E.; Panayotov, D.; Thompson, T. L.; Yates, J. T. Light-Induced Charge Separation in Anatase $\mathrm{TiO}_{2}$ Particles. J. Phys. Chem. B 2005, 109 (13), 6061-6068.

(56) Pham, H. H.; Wang, L.-W. W. Oxygen Vacancy and Hole Conduction in Amorphous $\mathrm{TiO}_{2}$. Phys. Chem. Chem. Phys. 2015, 17 (1), 541-550.

(57) Zhao, L.; Magyari-Köpe, B.; Nishi, Y. Polaronic Interactions between Oxygen Vacancies in Rutile $\mathrm{TiO}_{2}$. Phys. Rev. B: Condens. Matter Mater. Phys. 2017, 95 (5), No. 054104.

(58) Ji, Y.; Wang, B.; Luo, Y. Location of Trapped Hole on Rutile$\mathrm{TiO}_{2}$ (110) Surface and Its Role in Water Oxidation. J. Phys. Chem. C 2012, 116 (14), 7863-7866.

(59) Li, W.; Sun, Y. Y.; Li, L.; Zhou, Z.; Tang, J.; Prezhdo, O. V. Control of Charge Recombination in Perovskites by Oxidation State of Halide Vacancy. J. Am. Chem. Soc. 2018, 140 (46), 15753-15763. (60) Wang, H.; Yong, D.; Chen, S.; Jiang, S.; Zhang, X.; Shao, W.; Zhang, Q.; Yan, W.; Pan, B.; Xie, Y. Oxygen-Vacancy-Mediated Exciton Dissociation in $\mathrm{BiOBr}$ for Boosting Charge-Carrier-Involved Molecular Oxygen Activation. J. Am. Chem. Soc. 2018, 140 (5), 17601766.

(61) Glass, D.; Cortés, E.; Ben-Jaber, S.; Brick, T.; Quesada-Cabrera, R.; Peveler, W. J. W. J.; Zhu, Y.; Blackman, C. S. C. S.; Howle, C. R.; Parkin, I. P. I. P.; Maier, S. A. S. A. Photo-Induced Enhanced Raman Spectroscopy (PIERS): Sensing Atomic-Defects, Explosives and Biomolecules. In Chemical, Biological, Radiological, Nuclear, and Explosives (CBRNE) Sensing XX; Guicheteau, J. A., Howle, C. R., Eds.; SPIE, 2019; Vol. 11010, pp 72-81. 